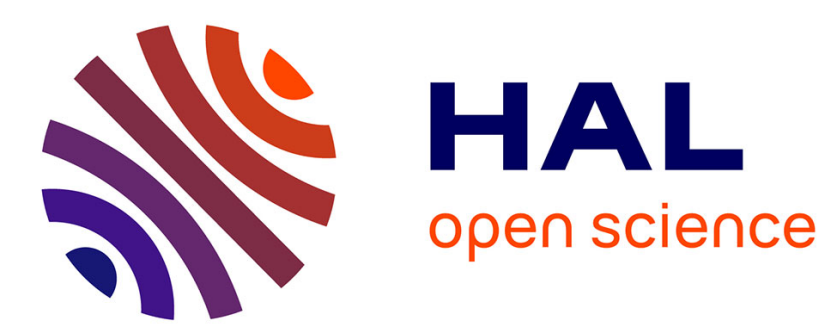

\title{
Design and optimization of three-dimensional extrusion dies, using constraint optimization algorithm
}

\author{
Nadhir Lebaal, Fabrice Schmidt, Stephan Puissant
}

\section{To cite this version:}

Nadhir Lebaal, Fabrice Schmidt, Stephan Puissant. Design and optimization of three-dimensional extrusion dies, using constraint optimization algorithm. Finite Elements in Analysis and Design, 2009, 45 (5), pp.333-340. 10.1016/j.finel.2008.10.008 . hal-01716280

\author{
HAL Id: hal-01716280 \\ https://hal.science/hal-01716280
}

Submitted on 15 Feb 2019

HAL is a multi-disciplinary open access archive for the deposit and dissemination of scientific research documents, whether they are published or not. The documents may come from teaching and research institutions in France or abroad, or from public or private research centers.
L'archive ouverte pluridisciplinaire HAL, est destinée au dépôt et à la diffusion de documents scientifiques de niveau recherche, publiés ou non, émanant des établissements d'enseignement et de recherche français ou étrangers, des laboratoires publics ou privés. 


\title{
Design and optimization of three-dimensional extrusion dies, using constraint optimization algorithm
}

\author{
Nadhir Lebaal ${ }^{\mathrm{a}, *}$, Fabrice Schmidt ${ }^{\mathrm{b}}$, Stephan Puissant ${ }^{\mathrm{a}}$ \\ ${ }^{a}$ GIP-InSIC, ERMeP, Institut Supérieur d'Ingénierie de la Conception, 27 Rue d'Hellieule, 88100 Saint-Dié, France \\ ${ }^{\mathrm{b}}$ Ecole des mines d'Albi Carmaux, Laboratoire CROMeP, Campus Jarlard- Route de Teillet, 81013 Albi Cedex 9, France
}

\begin{abstract}
A B S T R A C T
Balancing the distribution of flow through a die to achieve a uniform velocity distribution is the primary objective and one of the most difficult tasks of extrusion die design. If the manifold in a Coat-hanger die is not properly designed, the exit velocity distribution may be not uniform; this can affect the thickness across the width of the die. Yet, no procedure is known to optimize the coat hanger die with respect to an even velocity profile at the exit. While optimizing the exit velocity distribution, the constraint optimization algorithm used in this work enforced a limit on the maximum allowable pressure drop in the die; according to this constraint we can control the pressure in the die. The computational approach incorporates three-dimensional finite element simulations software Rem3D ${ }^{\circledR}$ and includes an optimization algorithm based on the global response surfaces with the Kriging interpolation and SQP algorithm within an adaptive strategy of the search space to allow the location of the global optimum with a fast convergence. The optimization results which represent the best die design are presented according to the imposed constraint on the pressure.
\end{abstract}

Keywords:

Polymer extrusion

Response surface method

DoE

Optimization

Kriging interpolation

Finite element analysis

\section{Introduction}

The design of dies for polymer extrusion often involves trial and error corrections of the die geometry to achieve uniform flow at the exit. If the repartition channel in a flat die is not designed properly, the velocity at the exit of the flat die may not be uniform [1], and leads to a variation in the sheet thickness across the width of the die.

Often, the number of the involved variables and their interactions prevent any optimization according to the trial and error corrections, because the number of evaluations needed may become very high. Design of experiment, in particular the Taguchi method [2], allows obtaining invaluable information on the important variables of the process in order to achieve the required goals. The effects of the various factors can be represented on graphs to support the discussion and to lead to identify the most sensitive to minimize the defects. Within this framework, we can mention Chen et al. [3]. They showed, using the Taguchi method, that the operating conditions, the type of materials, and the geometry of the die have a great influence on the exit velocity distribution on the die.

Prior works in sheet die optimization have involved the use of lubrication approximations of the momentum equations [4,5]. If the

\footnotetext{
* Corresponding author. Tel.: +33329421 821; fax: +33329421825.

E-mail addresses: lebaal_nadir@yahoo.fr, nadhir.lebaal@insic.fr (N. Lebaal).
}

geometry is more complex, a flow channel can be approximated with simple geometric sections [6]. Smith et al. [7,8] modeled Newtonian and non-Newtonian isothermal flow in a coat hanger die using a generalized Hele-Shaw (HS) approximation, and optimized the die by minimizing pressure drop subject to exit flow uniformity being within a tolerance set. The sensitivities analysis needed for the sequential quadratic programming (SQP) algorithm was calculated by direct differentiation and the adjoint method are compared, and simultaneous minimization of velocity dispersion subject to residence time variations is added using Broyden Fletcher Goldfarb Shanno (BFGS) algorithm and penalty function. The same author [9] in order to optimize the shape of the extrusion die for two different materials at various temperatures, used and compared two optimization algorithms with constraint based on SQP and sequential linear programming (SLP). The optimization problem consists in minimizing the pressure loss in the die, with an imposed constraint so that a homogeneous velocity distribution is obtained on the outlet side of the die within an imposed tolerance. Network algorithms have been developed to optimize die designs [10] but they are difficult to apply to arbitrary shapes. Michaeli et al. [11] have used a combination of finite element analysis for isothermal flow and flow analysis network to accelerate the iterative optimization process for the design of profile extrusion dies. To optimize the die geometry, they used, respectively, the evolution strategy algorithm and network theory. Sun et al. [12] optimize a flat die using BFGS algorithm. A penalty 


\begin{tabular}{|c|c|c|c|}
\hline \multicolumn{2}{|l|}{ Nomenclature } & $J$ & normalized objective function \\
\hline$a, m, \tau, A_{1}, A_{2}$ & material constants & $\widetilde{J}(x)$ & objective or constraint interpolate \\
\hline$\hat{a} \beta$ & coefficients vectors & & function \\
\hline$\alpha$ & weight coefficient & $k$ & number of the basis function in re- \\
\hline$A, B, C, D$ & optimization variables & & gression model \\
\hline$c$ & dilation parameter & $N$ & number of nodes at the die exit \\
\hline$d_{i}$ & $\begin{array}{l}\text { distance from a discrete node } x_{i} \text { to } \\
\text { a sampling point } x\end{array}$ & $\begin{array}{l}P \\
P_{0}\end{array}$ & $\begin{array}{l}\text { pressure } \\
\text { initial pressure in the die }\end{array}$ \\
\hline E & velocity dispersion & $\hat{p}(x)$ & basis function \\
\hline$E_{0}$ & initial velocity dispersion & $R$ & correlation matrix \\
\hline$\varepsilon(v)$ & strain rate tensor & $r_{w}$ & radius of support domain \\
\hline$\widetilde{\varepsilon}$ & tolerance & $S 1, S 2, S 3$ & surfaces \\
\hline$F(x)$ & responses from the function & $T$ & temperature \\
\hline$g$ & constraint function & $T_{\text {ref }}$ & references temperature \\
\hline$\dot{\bar{\gamma}}$ & shear rate & $v$ & velocity \\
\hline$\eta$ & fluid viscosity (dependent of the & $v_{i}$ & exit velocity \\
\hline$T$ & temperature $T$, pressure $p$, and of & $\bar{v}$ & average exit velocity \\
\hline & the strain rate tensor $\varepsilon(v)$ through & $w_{i}(x)$ & weight function of Gaussian type \\
\hline & the shear rate $\overline{\bar{\gamma}}$ ) & & design variables \\
\hline$\eta_{0}(T)$ & thermal dependency & $Z(x)$ & random fluctuation \\
\hline
\end{tabular}

function was introduced to enforce a limit on the maximum allowable pressure drop in the die.

The optimization algorithm must be carefully chosen when one single analysis using three-dimensional software requires several hours of CPU time. Non-deterministic or stochastic methods such as Monte Carlo method and genetic algorithm [13] can obtain global minimum but they need a lot of evaluations for the functions to converge. Gradient methods [7-10,12,14] require the computations of the gradients of the functions; the computation of gradients by finite difference is time consuming and depends on the perturbed parameters. For the above reasons we decided to chose a response surface method (RSM) [15].

The ultimate goal of this work is to optimize the coat hanger sheet die geometry (Fig. 1) in a way that a uniform velocity distribution is obtained at the die exit with an imposed nonlinear constraint so that the pressure loss in the die must decrease compared to the initial die.

For this end, we developed an automatic optimization algorithm based on SQP algorithm and a RSM together with Kriging interpolation and several strategies to permit to obtain a precise global optimum with a fast convergence. A preliminary study based on Taguchi's design of experiments method [3] was conducted, in order to identify the most sensitive design variables. To compute a 3D flow in extrusion dies we used FEM software (REM3D $\left.{ }^{\circledR}\right)$ [16]. This software takes into account strain rate and temperature dependence.

\section{Modeling and simulation}

The extrusion simulation is carried out using the 3D computation software by finite elements REM3D ${ }^{\circledR}[16]$.

The flow equations are derived from the Navier-Stokes incompressible equations. A mixed finite element method for incompressible viscous flow is used. The flow solver uses tetrahedral elements with a linear continuous interpolation of both the pressure and the velocity and a bubble enrichment of velocity.

The mass, momentum and energy conservation equations, are used to follow the material behavior, from which the velocity, pressure and temperature fields are determined.

$$
\left\{\begin{array}{l}
\nabla(2 \eta(\dot{\bar{\gamma}}) \dot{\varepsilon}(v))-\nabla p=0 \\
\nabla \cdot \vec{v}=0 \\
\rho \hat{c} \frac{\mathrm{d} T}{\mathrm{~d} t}=-\nabla \cdot q+\sigma: \dot{\varepsilon}(v)
\end{array}\right.
$$

The behaviors laws used in Rem3D ${ }^{\circledR}$ give an expression of the viscosity in function of the shear rate and temperature. In this paper, the geometry of a flat die is optimized for an acrylonitrile butadiene styrene (ABS, Astalac EPC 10000). The rheological parameters of the ABS are given in Table 1. Carreau Yasuda/WLF viscosity model is used to characterize the temperature and shear rate dependence [17]. It is written as

$\eta=\eta_{0}(T)\left[1+\left(\eta_{0}(T) \frac{\dot{\bar{\gamma}}}{\tau_{s}}\right)^{\alpha}\right]^{m-1 / \alpha}$

In this model, $a, m, \tau$ are material constants, whereas $\eta_{0}(T)$ establishes the thermal dependency, given by the WLF model:

$\eta_{0}(T)=\eta_{0}\left(T_{r e f}\right) \exp \left[\frac{A_{1}\left(T_{r e f}-T_{S}\right)}{A_{2}+\left(T_{r e f}-T_{S}\right)}-\frac{A_{1}\left(T-T_{S}\right)}{A_{2}+\left(T-T_{S}\right)}\right]$

where $A_{1}, A_{2}$ are material constants, and $T_{\text {ref }}$ is the references temperature.

A flow of $50000 \mathrm{~mm}^{3} / \mathrm{s}$ was imposed on the entry with a temperature of $240^{\circ} \mathrm{C}$, and the temperature of the die is constant and equals to $230^{\circ} \mathrm{C}$.

\section{Formulation of the optimization problem}

\subsection{Objective and constraint functions}

This optimization problem consists in determining an optimal geometry to homogenize the velocity distribution through the die exit, which corresponds to the minimum of the velocity dispersion $(E)$. While preventing that the pressure "pressure loss" increases more than the pressure obtained by the initial geometry. We can also impose a more severe constraint on the pressure; this condition is translated by a constraint function $(g)$.

$$
\begin{cases}\min & J(\Phi)=\frac{E}{E_{0}} \\ \text { such that } & g=\frac{P-\left(\alpha * P_{0}\right)}{\left(\alpha * P_{0}\right)} \leqslant 0\end{cases}
$$

where $(J)$, the normalized objective function, the velocity dispersion $(E)$, is defined as

$$
E=\left(\frac{1}{N} \sum_{i=1}^{N}\left(\frac{\left|v_{i}-\bar{v}\right|}{\bar{v}}\right)\right)
$$




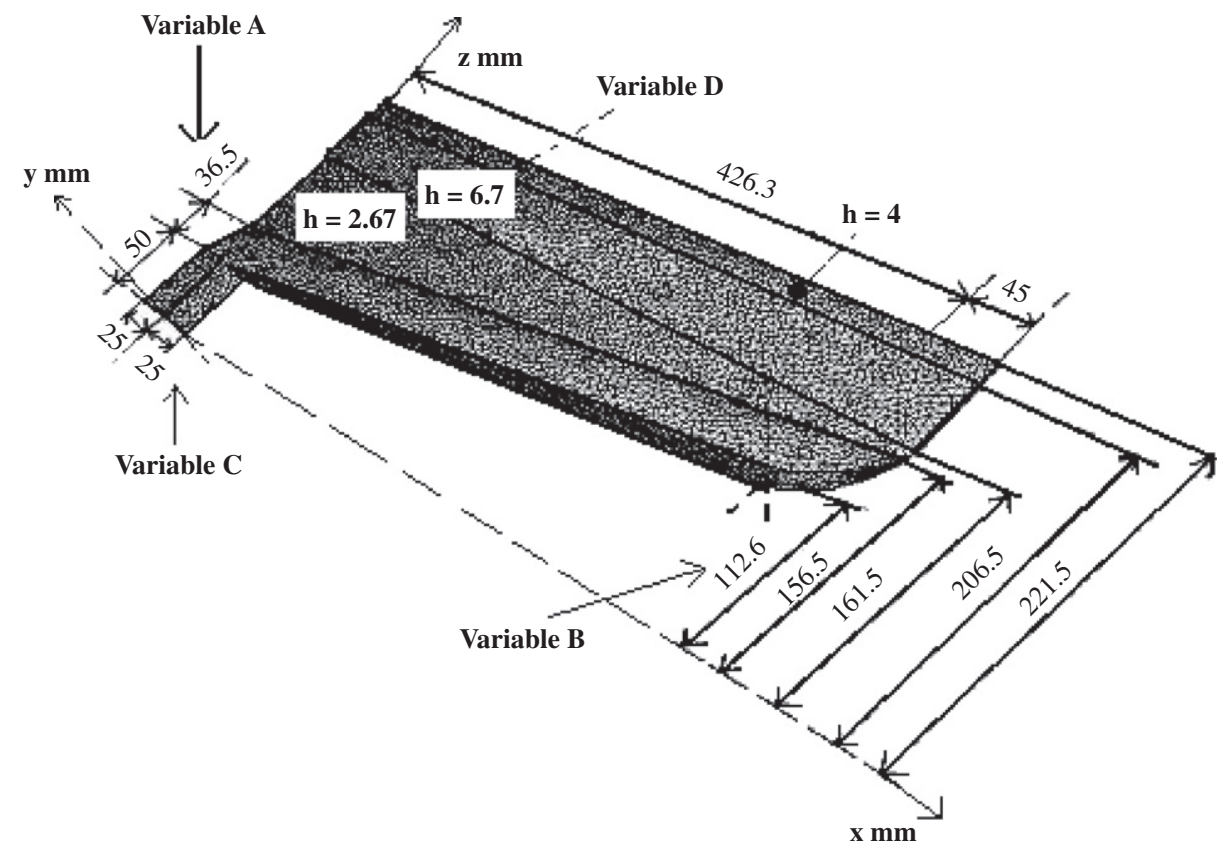

Fig. 1. Geometry of the flat die.

Table 1

Rheological parameters

\begin{tabular}{llll}
\hline$\eta_{0}$ (Pa s) & $m$ & $\alpha_{0}$ & $\tau_{s}(\mathrm{~Pa})$ \\
\hline 716.997 & 0.15862 & 1 & 224063 \\
$A_{1}$ & $A_{2}(\mathrm{~K})$ & $T_{s}(\mathrm{~K})$ & $T_{\text {ref }}(\mathrm{K})$ \\
\hline 20.4 & 101.6 & 397.7 & 524.7 \\
\hline
\end{tabular}

where $E_{0}$ and $P_{0}$ are, respectively, the velocity dispersion and the pressure in the initial die, $N$ is the total number of nodes at the die exit in the middle plane, $v_{i}$ is the velocity at an exit node, and $\bar{v}$ is the average exit velocity, is defined as

$\bar{v}=\frac{1}{N} \sum_{i=1}^{N} v_{i}$

The constraint function $(g)$ is selected in a way that it is negative if the pressure is lower than the imposed pressure, if not it will be positive. If $\alpha=1$; the pressure should not increase compared to the initial pressure. If $\alpha<1$; the pressure must be even lower compared to the initial pressure.

\subsection{Design variables}

The design of a flat die is based on the values of various geometrical parameters; the optimization variables $(A, B, C$ and $D)$ correspond to the geometry of the coat hanger die. The first variable $A$ represents the depth of the channel repartition, the second variable $B$ represents the opening of the channel repartition, the third one $C$ represents the channel thickness and the fourth variable $D$ represents the thickness of the relaxation zone (Fig. 1). During the optimization process the variables $A, B, C$ and $D$ vary in a limited field. Each variable has its geometrical limitations which are, respectively, $30 \leqslant A \leqslant 110 \mathrm{~mm}$, $50 \leqslant B \leqslant 140 \mathrm{~mm}, 5 \leqslant C \leqslant 45 \mathrm{~mm}$ and $6.7 \leqslant D \leqslant 15 \mathrm{~mm}$.

Initially, the four geometrical variables of the die are $A=36.5 \mathrm{~mm}$, $B=112.6 \mathrm{~mm}, C=25 \mathrm{~mm}$ and $D=6.7 \mathrm{~mm}$.

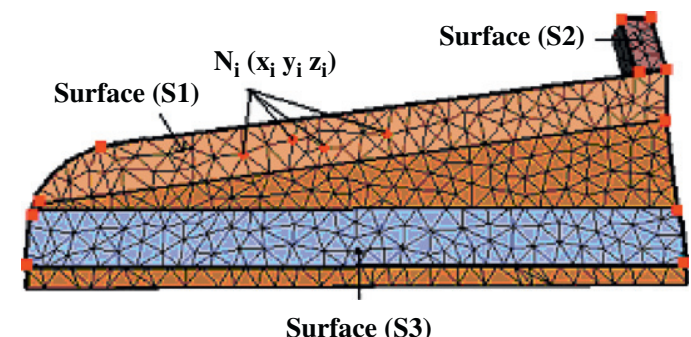

Fig. 2. Procedure for the change of the geometry.

During the optimization process, we developed a Matlab ${ }^{\circledR}$-based program which, allows us to treat the CAD and to change the geometry of the die automatically. In the first stage, in order to change the geometry we make a surfaced mesh. Since we know the nodes position, we seek to determine the coordinates of all the nodes $\left(N_{i}\right)$, belonging to both surfaces $S 1, S 2$ and $S 3$ (Fig. 2). For this, we use the diffuse approximation [14] with a linear interpolation.

To change the geometry of the channel repartition we define the new surface $\left(S 1_{k}\right)$, which corresponds to the new optimization parameters $A_{k}, B_{k}$, and $C_{k}$. After that, we carry out a change of coordinates for all nodes which belong to the surface $S 1$, so that they will be relocated on to the new $S 1_{k}$ surface which represents the new geometry. For $S 2$ and $S 3$ surface, a simple translation of the following nodes is applied there. The same procedure is applied on the other side of the die.

\subsection{Optimization procedure}

The objective and constraint function (Eq. (4)) are implicit compared to the optimization parameters and its evaluation need a complete numerical analysis of the extrusion process, which requires a large computation times. To need the optimum parameters with low cost and with a good accuracy, the RSM is adopted and coupled with an auto adaptive strategy of the research space Fig. 3. The RSM consists in the construction of an approximate expression of objective and constraint function starting from a limited number of evaluations of the real function. In order to obtain a good 


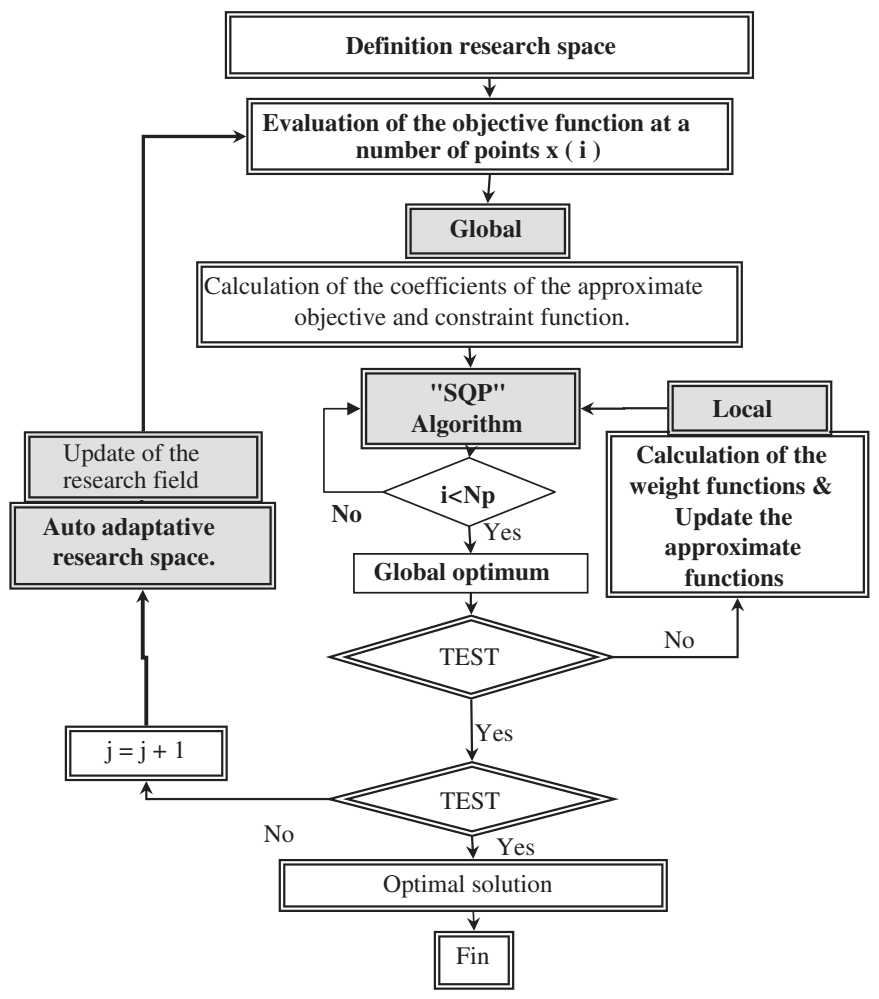

Fig. 3. The flowchart of the adopted optimization strategy.

approximation, we used a Kriging interpolation described in next section. In this method, the approximation is computed by using the evaluation points by composite design of experiments.

Once the approximation of the objective function and the constraint function are built for each iteration. Since the successive evaluation of the approximate function does not take much computing time, we use the SQP algorithm to obtain the optimal approximate solution which respects the imposed nonlinear constraints. To avoid the fact of falling into a local optimum, and to respect the imposed nonlinear constraint, we use an automatic procedure which allows the launch of the SQP algorithm starting from each point of our experimental design (Fig. 3). We take then the best approximate solution among those obtained by the various optimizations. Then, another approximate function is built by taking into account the weight function of Gaussian type which allows to slightly change the interpolation and makes the approximation more accurate locally (centred around the best minimum). Next, another minimization is carried out by the SQP algorithm with the initial point which represents the best optimum The iterative procedure stops when the successive points of the approximate function are superposed with a tolerance $\widetilde{\varepsilon}=10^{-6}$. Finally, another evaluation is carried out to obtain the real response in the optimization iteration.

An adaptive strategy of the search space is applied to allow the location of the global optimum and, then, to readjust the research space by decreasing it by $\frac{1}{3}$. A new design of experiment is automatically launched around the optimum. An enrichment of the interpolation is made by recovering responses already calculated, and which are located in the new research space. The iterative procedure stops when the successive points are superposed with a tolerance $\widetilde{\varepsilon}=10^{-3}$.

\subsection{Kriging interpolation}

The Kriging interpolation $[18,19]$, makes it possible to present the complexes function effectively. This method is applied in our work to represent the response surface in an explicit form, according to the variables of optimization. The approximate explicit relationship of the objective and constraint function, can be expressed as follows:

$\widetilde{J}(x)=\hat{p}^{\mathrm{T}}(x) \hat{a}+Z(x)$

with $\hat{p}(x)=\left[\hat{p}_{1}(x), \ldots, \hat{p}_{k}(x)\right]^{\mathrm{T}}$, where $k$ denotes the number of the basis function in regression model, $\hat{a}=\left[\hat{a}_{1}, \ldots, \hat{a}_{k}\right]^{\mathrm{T}}$ is the coefficient vector the, $x$ is the design variables, $\widetilde{J}(x)$ is the unknown objective or constraint interpolate function, and $Z(x)$ is the random fluctuation. The term $\hat{p}^{\mathrm{T}}(x) \hat{a}$ in Eq. (7) indicates a global model of the design space, which is similar to the polynomial model in a moving least squares (MLS) approximation. The second part in Eq. (7) is a correction of the global model. It is used to model the deviation from $\hat{p}^{\mathrm{T}}(x) a$ so that the whole model interpolates response data from the function

The construction of a Kriging model can be explained as follows:

The output responses from the function are given as

$F(x)=\left\{f_{1}(x), f_{2}(x), \ldots f_{n}(x)\right\}$

From these outputs the unknown parameters $\hat{a}$ can be estimated:

$\hat{a}=\left(\hat{P}^{\mathrm{T}} R^{-1} \hat{P}\right)^{-1} \hat{P}^{\mathrm{T}} R^{-1} F$

where $\hat{P}$ is a vector including the value of $\hat{p}(x)$ evaluated at each of the design variables and $R$ is the correlation matrix, which is composed of the correlation function evaluated at each possible combination of the points of design:

$$
\begin{aligned}
R= & {\left[\begin{array}{ccc}
R\left(x_{1}, x_{1}\right) & \cdots & R\left(x_{1}, x_{n}\right) \\
\vdots & \ddots & \vdots \\
R\left(x_{n}, x_{1}\right) & \cdots & R\left(x_{n}, x_{n}\right)
\end{array}\right] } \\
& +\left[\begin{array}{cccc}
w\left(x-x_{1}\right) & 0 & \cdots & 0 \\
0 & w\left(x-x_{2}\right) & \cdots & 0 \\
\vdots & \vdots & \vdots & \vdots \\
0 & 0 & \cdots & w\left(x-x_{n}\right)
\end{array}\right] \\
R_{i j} & =\left(\left|x_{i}-x_{j}\right|\right)
\end{aligned}
$$

A weight function of Gaussian type with a circular support is adopted for the Kriging interpolation. It takes the form

$w_{i}(x)=\left\{\begin{array}{cc}\left(1-\frac{e^{-\left(d_{i} / c\right)^{2}}-e^{-\left(r_{w} / c\right)^{2}}}{1-e^{-\left(r_{w} / c\right)^{2}}}\right) & \text { si } d_{i} \leqslant r_{w} \\ 1 & \text { si } d_{i} \geqslant r_{w}\end{array}\right.$

where $d_{i}=\sqrt{\sum_{J=1}^{n}\left(x^{J}-x_{(i)}^{J}\right)^{2}}$ is the distance from a discrete node $x_{i}$ to a sampling point $x$ in the domain of support with radius $r_{w}$, and $c$ is the dilation parameter. $c=r_{w} / 4$ is used in computation.

The second part in Eq. (7) is in fact an interpolation of the residuals of the regression model $\hat{p}^{\mathrm{T}}(x) \hat{a}$. Thus, all response data will be exactly predicted; is given as

$Z(x)=r^{\mathrm{T}}(x) \beta$

where $r^{\mathrm{T}}(x)=\left\{R\left(x, x_{1}\right), \ldots, R\left(x, x_{n}\right)\right\}$.

The parameter $\beta$ is defined as follows:

$\beta=R^{-1}(F-\hat{P} \hat{a})$

\section{Result and discussion}

\subsection{Design sensitivity analysis}

A Taguchi's design of experiments method [3] is used to investigate the sensitive analysis of the design variables. This preliminary 


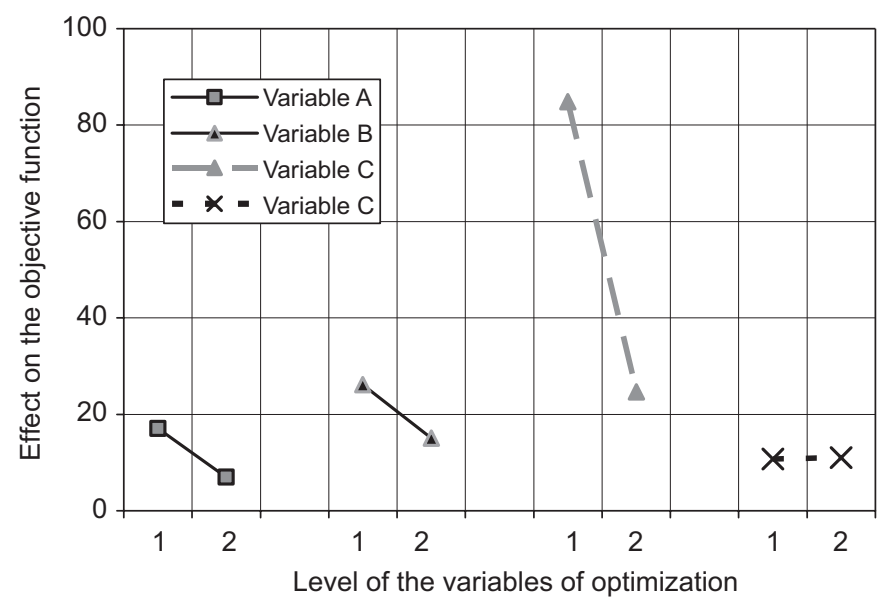

Fig. 4. Effect of the variables on the objective function.

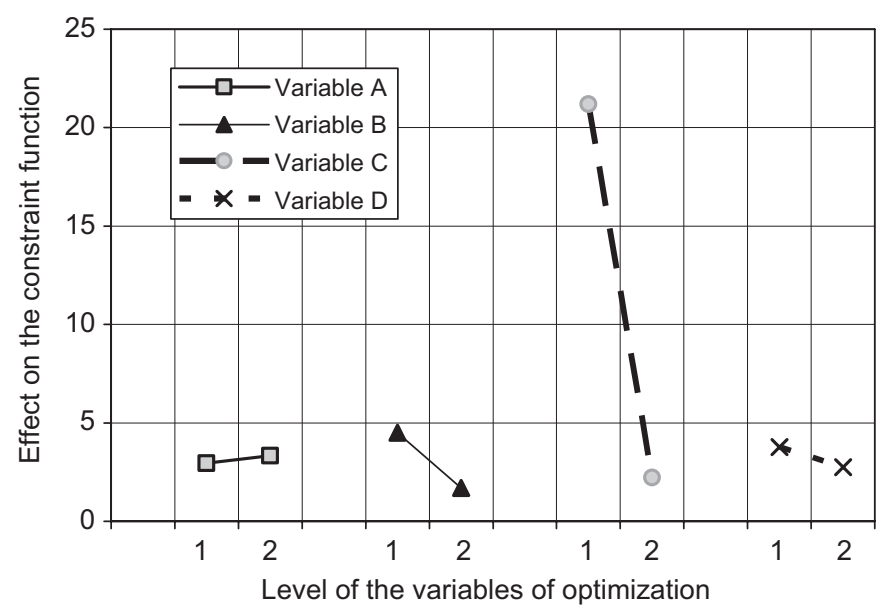

Fig. 5. Effect of the variables on the constraint function.

study was conducted, by considering four geometrical parameters $(A, B, C$ and $D)$, in order to identify the most sensitive ones on the objective and the constraint function.

For this, we have calculated the effect of each variable over all the field of research by studying the contribution of each variable on the variation of the response "velocities distribution and pressure". The effects of the various variables are represented on graphs to support the discussion and to lead to the identification of those influencing to minimize the defects.

We note in Fig. 4 that the variable with less influence on the objective function is the variable $D$ which represents the thickness of the relaxation zone. On the other hand, the variable $C$ has the greatest effect on the velocity distribution at the die exit.

We observe in the seam figure that if the variables " $A, B$, and $C$ " are in the lowest level (1), the global relative variation is more important which implies a bad velocity distribution at the die exit. On the other hand, if the variables are in the highest level (2), the global relative variation of velocity at the die exit is less important compared to their lowest level.

For the variable " $C$ ", it is even weaker at the average level, and for the variable " $D$ ", it is noted that it has a very weak effect over the velocity distribution at the die exit.

From Fig. 5 we note that the effect of the variables $B$ and $D$ on the constraint function (pressure) is weak. We note that the more the variables " $A, C$ and $D$ " are in the lower level the more the pressure increases. On the other hand, the pressure is lower at their highest level. For the variable $B$, the variation of the pressure is very weak.

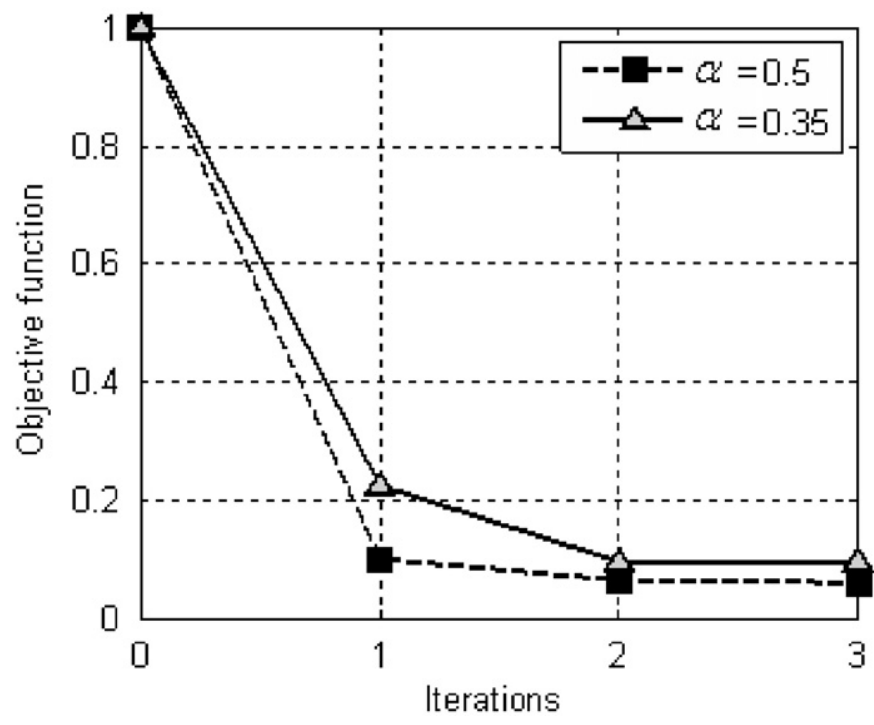

Fig. 6. Convergence history during an optimization process on the objective functions.

Table 2

Summary of the results

\begin{tabular}{llll}
\hline Results of optimization & Initial die $(A)$ & $\begin{array}{l}\text { Result 1 } \\
\alpha=0.5(B)\end{array}$ & $\begin{array}{l}\text { Result 2 } \\
\alpha=0.35(C)\end{array}$ \\
\hline "J" & 1 & 0.049 & 0.09 \\
Improvement (\%) & - & 95 & 91 \\
"g" & 1 & 0.48 & 0.38 \\
Profit of pressure (\%) & - & 52 & 62 \\
Variable $A(\mathrm{~mm})$ & 36.5 & 96.6 & 103.08 \\
Variable B (mm) & 112.6 & 76.15 & 72.74 \\
Variable C (mm) & 25 & 25.47 & 31.4 \\
\hline
\end{tabular}

Since the prime objective in the design of the extrusion dies is to obtain at the exit a perfectly homogeneous product, it was noted that the variable $D$ has the least important effect on the velocity distribution at the die exit. This study enables us to limit the number of variables and three sensitive geometrical parameters are selected and considered as design variables to be optimized $(A, B$ and $C)$.

\subsection{Optimization results}

The optimization strategy described previously is applied to optimize the die geometry in order to obtain a homogenous exit velocities distribution. According to the imposed constraint on the pressure, two optimization cases are formulated:

Case 1: A constraint on the pressure is imposed for which the pressure in the optimal die must be lower or equals $0.5 * P_{0}(\alpha=0.5)$.

Case 2: The constraint imposed on the pressure is more severe. The pressure in the optimal geometry must be lower or equals $0.35 * P_{0}(\alpha=0.35)$.

A representative convergence history during an optimization process on the objective functions $(J)$ is represented in Fig. 6. After three iterations, the objective function fell below the cutoff of $10^{-3}$ and the simulation stopped.

Given the fixed geometry constraints of the die, the rheological parameters of the ABS resin, and the process conditions, the optimal solution is obtained starting from the second iteration for both cases. The objective function is then reduced to $95 \%$ of its initial value for the first case $(\alpha=0.5)$ and $91 \%$ for the second case $(\alpha=0.35)$. The optimization strategy clearly showed its capacity to obtain an optimal solution with a very fast convergence. A summary of the optimization results obtained for the two cases, is reported in Table 2. 
We notice that the objective function for both cases has strongly decreased in the first iteration, because of the precision of the Kriging interpolation. Nevertheless, to respect the constraint while ensuring a homogeneous distribution of velocity, we observe that case 1 offer the best minimum, because of the less severe constraint on the pressure compared to case 2 .

We have represented the relative velocity dispersion obtained in the initial geometry and after optimization, for both results in Fig. 7. We see that the objective function obtained by imposing a severe constraint on the pressure $(\alpha=0.35)$ is slightly higher of the objective function obtained with a less severe constraint $(\alpha=0.5)$.

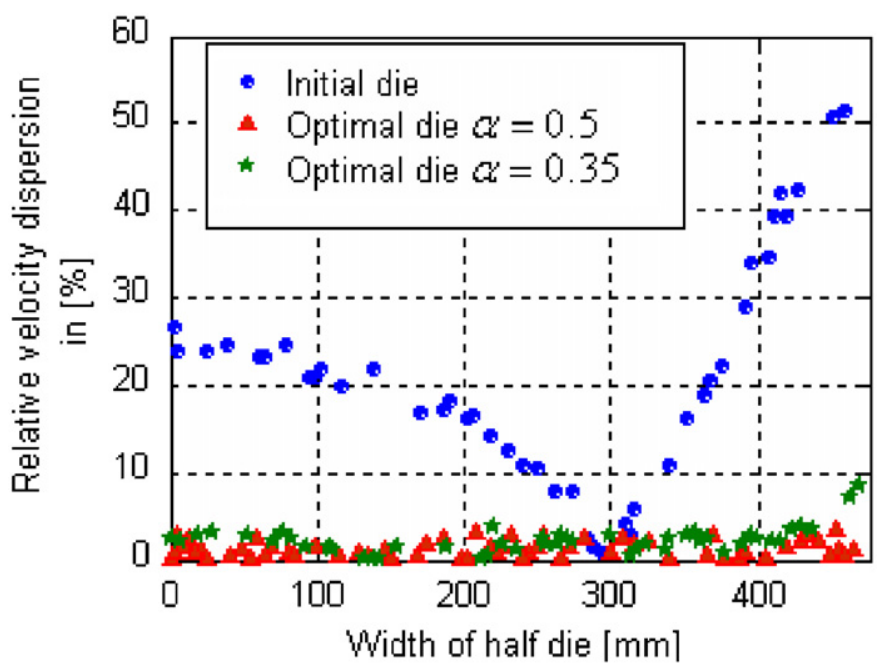

Fig. 7. Velocity dispersion with initial and optimal die.
For a qualitative comparison it is obvious that velocity distribution in the initial geometry (Fig. 8A) is not homogeneous. We notice that in the middle of the die, velocities are lower about $35 \%$ of variation compared to the average. On the other hand, in both parts close to the end of the die, we observe an increase of velocities until $50 \%$ (Fig. 7), only in the small regions near the end of this die, the exit velocity reduces to zero to satisfy the no slip condition at the walls. After optimization, we notice that velocities became homogeneous all over the width at the die exit. For both optimization results, seeing, respectively, Fig. 8B and C.

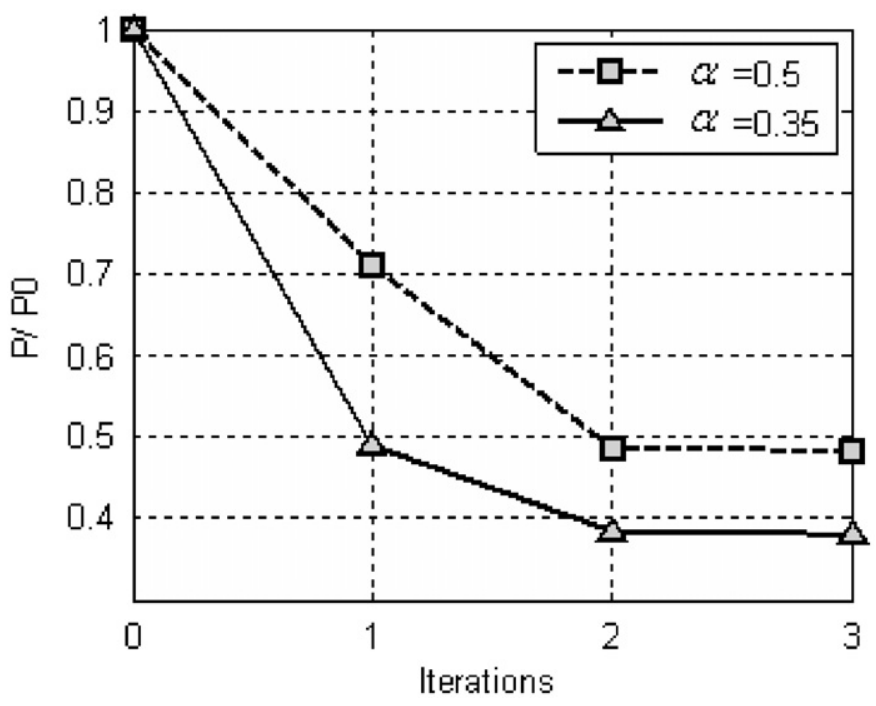

Fig. 9. Convergence history of the constraint functions during an optimization run.

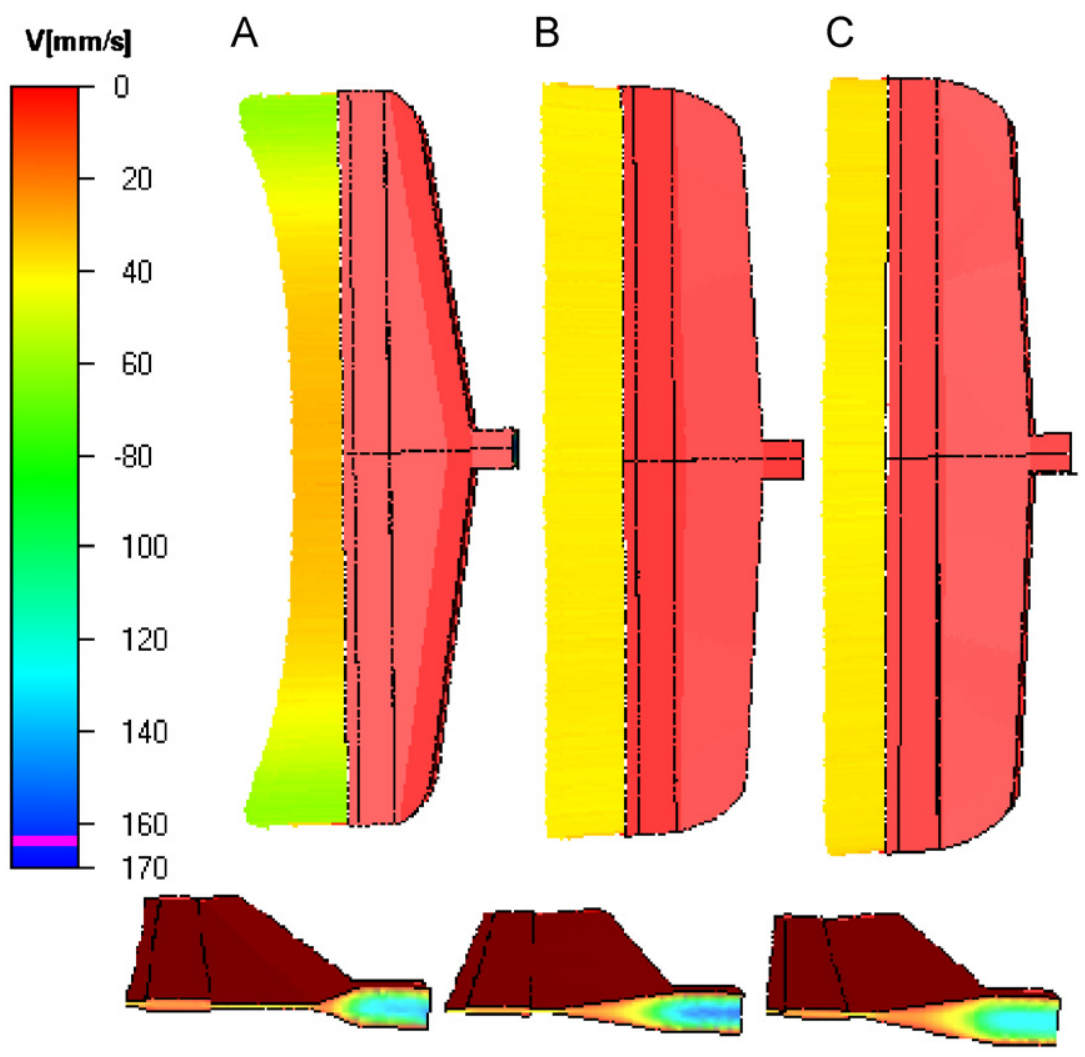

Fig. 8. Velocity distribution in initial and optimal dies. 
A

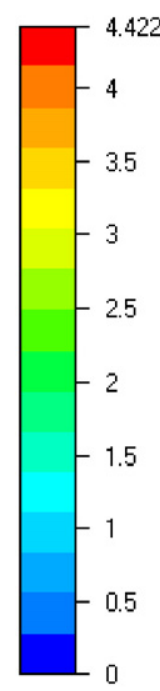

B

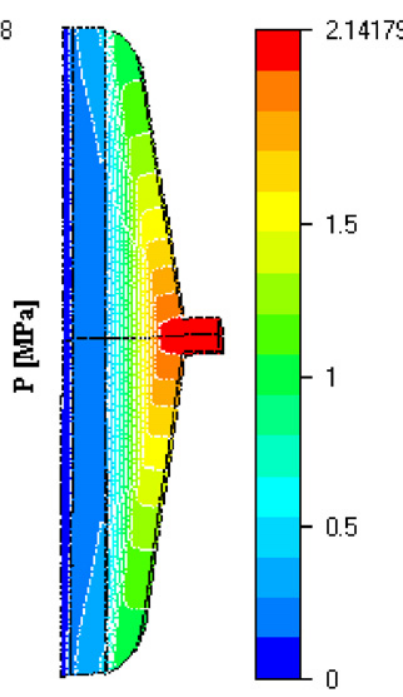

C

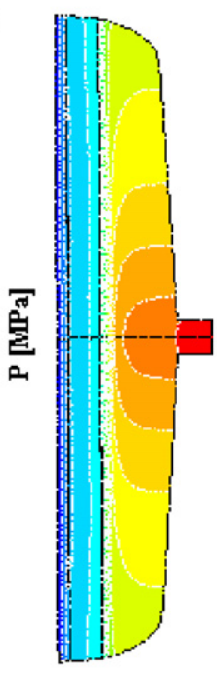

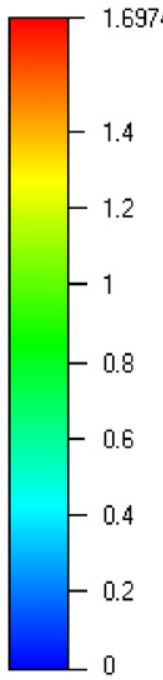

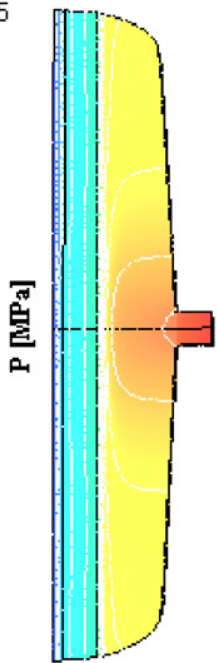

Fig. 10. Pressure distribution in initial and optimal dies.

A

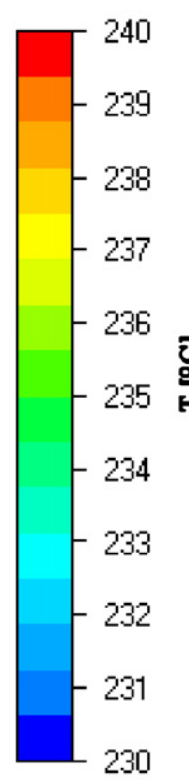

B

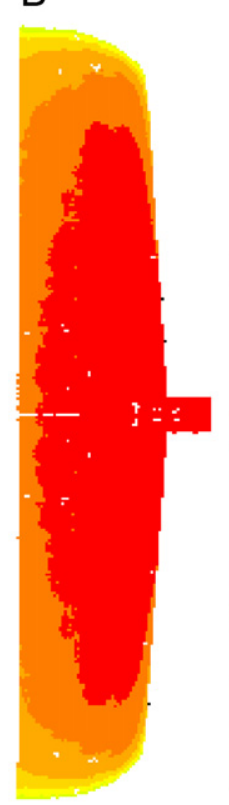

C

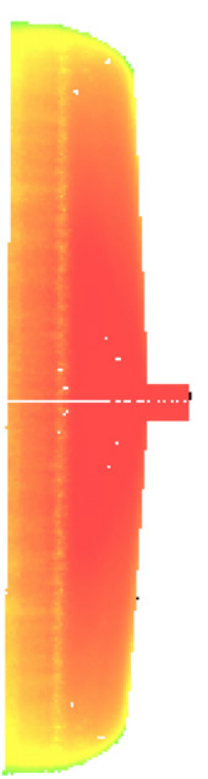

Fig. 11. Temperature distribution in mid plane in initial and optimal dies.

The constraint on the pressure is represented on Fig. 8, in a form of $\left(P / P_{0}\right)$ for both cases. We observe that the constraint function is weaker with a more severe constraint $(\alpha=0.35)$.

This optimization problem was formulated in a way to find the best velocity distribution at the die exit while avoiding the increase of pressure compared to an imposed pressure, which is respected by both results (Fig. 9). The pressure drop in the design of the initial die is $4.42 \mathrm{MPa}$ (Fig. 10A). It should be noted that the pressure decreases during the iterations of optimization for the different results. In the first case, the pressure decreases by $52 \%$ which gives a pressure of $2.14 \mathrm{MPa}$ (Fig. 10B). In the results of the second case, we note that the pressure has decreased by $62 \%$ because of the imposed constraint (Fig. 9), which gives a pressure of $1.69 \mathrm{MPa}$ (Fig. 10C). We notice that the gradient pressure in the initial die is not parallel, which results on a non-uniform flow. Nevertheless, in the optimized dies

the gradient pressure is parallel; furthermore, it denotes a uniform flow distribution for both cases.

It is noted that more the polymer runs towards the border because of the heating by shearing, the temperature is lower in the middle of the die and higher close to the border (Fig. 11A). By comparison, the distribution of the temperatures is uniform in the optimal die (Fig. 11B and C), because of the homogeneous velocities distribution (due to the heating by shearing) at the die exit for the optimal geometries.

\section{Conclusion}

The design of polymer extrusion dies is complicated by the nonlinear relationship between the resin viscosity, shear rate and temperature. The first study based on the sensitive analysis enabled us to decrease the number of variables by analyzing their effects on the pressure and the velocity distribution at the die exit. The numerical optimization algorithm presented in this work shows its robustness as a tool for extrusion die design. The applied optimization method with the various strategies proves its ability of predicting optimal die geometry with satisfactory computing time for three-dimensional finite element calculation.

The obtained results with the two optimization examples show that our optimization strategy is well adapted to the nonlinear constraints. The use of the different constraints on the pressure allowed us to compare the various results obtained after optimization. The optimal die gives a good exit velocity distribution while minimizing the pressure for the same flow. A qualitative comparison enables us to note that we were able to obtain with the optimal geometry a $95 \%$ profit of homogeneity compared to the initial geometry with a decrease of pressure of $52 \%$ (results obtained with a less severe constraint on the pressure). The algorithm of optimization clearly showed its robustness and its capacity to obtain an optimal solution with a fast convergence.

Future research will involve experimental verification of the algorithm and the optimization of complex helicoidally dies.

\section{References}

[1] Y. Wang, The flow distribution of molten polymers in slit dies and coat hanger die through three-dimensional finite element analysis, Polym. Eng. Sci. 31 (1991) 204-212. 
[2] D.C. Montgomery, Design and Analysis of Experiments, John Wiley \& Sons, Inc, USA, 2005

[3] C. Chen, P. Jen, F.S. Lai, Optimization of the coat hanger manifold via computer simulation and orthogonal array method, Polym. Eng. Sci. 37 (1997) $188-196$.

[4] Y.W. Yu, T.J. Liu, A simple numerical approach for the optimal design of an extrusion die, J. Polym. Res. 5 (1998) 1-7.

[5] Y. Xiaorong, S. Changyu, L. Chuntai, W. Lixia, Optimal design for polymer sheeting dies, Chines J. Comput. Mech. 21 (2004) 253-256.

[6] S. Puissant, Y. Demay, B. Vergnes, Two-dimensional multilayer coextrusion flow in a flat coat-hanger die. Part I: modeling, Polym. Eng. Sci. 34 (1994) 201-208.

[7] D.E. Smith, D.A. Tortorelliav, C.L. Tucker, Optimal design for polymer extrusion. Part I: sensitivity analysis for nonlinear steady-state systems, Comput. Methods Appl. Mech. Eng. (1998) 283-302.

[8] D.E. Smith, D.A. Tortorelliav, C.L. Tucker, Optimal design for polymer extrusion. Part II: sensitivity analysis for weakly-coupled nonlinear steady-state systems, Comput. Methods Appl. Mech. Eng. (1998) 303-323.

[9] D.E. Smith, An optimisation-based approach to compute sheeting die designs for multiple operating conditions, SPE ANTEC Technical Papers, 2003, pp. 315-319.

[10] P. Hurez, P.A. Tanguy, D. Blouin, A new design procedure for profile die, Polym. Eng. Sci. 36 (1996) 626-635.
[11] W. Michaeli, S. Kaul, T. Wolff, Computer aided optimisation of extrusion dies, J. Polym. Eng. 21 (2001) 225-237.

[12] Y. Sun, M. Gupta, Optimization of a flat die geometry, SPE ANTEC Technical Papers, 2004, pp. 3307-3311.

[13] W. Michaeli, S. Kaul, Approach of automatic extrusion die optimisation, J. Polym. Eng. 24 (2004) 123-136.

[14] H.J. Ettinger, J. Sienz, J.F.T. Pittman, A. Polynkin, Parameterization and optimisation strategies for the automated design of U PVC profile extrusion dies, Struct. Multidisc. Optim. 28 (2004) 180-194.

[15] N. Lebaal, S. Puissant, F.M. Schmidt, Rheological parameters identification using in-situ experimental data of a flat die extrusion, J. Mater. Process. Tech. Papers 34 (2005) 1524-1529.

[16] L. Silva, Viscoelastic compressible flow and applications in 3D injection molding simulation, Thèse de Doctorat, CEMEF, 2004.

[17] G. Balasubrahman, D. Kazmer, Thermal control of melt flow in cylindrical geometries, SPE ANTEC Technical Papers, 2003, pp. 387-391.

[18] I. Kaymaz, Application of Kriging method to structural reliability problems, Struct. Safety 27 (2005) 133-151.

[19] H. Li, Q.X. Wang, K.Y. Lam, Development of a novel meshless local Kriging (LoKriging) method for structural dynamic analysis, Comput. Methods Appl. Mech. Eng. 193 (2004) 2599-2619. 